\title{
Erratum to: What is the preclinical evidence on platelet rich plasma and intervertebral disc degeneration?
}

\author{
Matteo Formica $^{1}$ - Luca Cavagnaroo ${ }^{1}$ Carlo Formica ${ }^{2}$ Maddalena Mastrogiacomo $^{3}$. \\ Marco Basso $^{1} \cdot$ Alberto Di Martino ${ }^{4}$
}

Published online: 13 October 2015

(c) Springer-Verlag Berlin Heidelberg 2015

\section{Erratum to: Eur Spine J}

DOI 10.1007/s00586-015-4189-2

There was an error in the authors name and their affiliation of this article.

The correct name is: Maddalena Mastrogiacomo.

DIMES, University of Genoa, IRCCS AOU San Martino-IST, Istituto Nazionale per la Ricerca sul Cancro, Largo Rosanna Benzi, 10, 16132 Genoa, Italy.

The online version of the original article can be found under doi:10.1007/s00586-015-4189-2.

Matteo Formica

dott.formica@gmail.com

1 Clinica Ortopedica, University of Genoa, IRCCS AOU San Martino-IST, Istituto Nazionale per la Ricerca sul Cancro, Largo Rosanna Benzi, 10, 16132 Genoa, Italy

2 CVCO, IRCCS Istituto Ortopedico Galeazzi, Via Riccardo Galeazzi, 420161 Milan, Italy

3 DIMES, University of Genoa, IRCCS AOU San MartinoIST, Istituto Nazionale per la Ricerca sul Cancro, Largo Rosanna Benzi, 10, 16132 Genoa, Italy

4 Department of Orthopaedics and Trauma Surgery, University Campus Bio-Medico of Rome, Via Álvaro del Portillo 21, Rome, Italy 